MATEC Web of Conferences 25,0300 5 (2015)

DOI: $10.1051 /$ matec conf/ 20152503005

(C) Owned by the authors, published by EDP Sciences, 2015

\title{
Application of EPON Technology in Transmission Line Video Monitoring
}

\author{
Zongze Xia, Fei Xia, Xiaobo Huang, Xiaoqing Li \& Ying Jia \\ State Grid Liaoyang Electric Power Supply Company, Liaoyang, Liaoning, China
}

\begin{abstract}
The operating condition of transmission lines directly determines the efficiency of the power system. Therefore, faced with a complex operating environment, it is extremely important to protect transmission line video monitoring. At present, the technology widely used in the power distribution and network communication in domestic power industry is EPON technology. This technology has a broad application prospect on the transmission of electrical circuit video monitoring information. On this basis, this paper carries out a further research on the application of EPON technology in transmission line video monitoring. This paper firstly proposes the design principle of transmission line video monitoring, and on this basis, it carries out a comparative analysis of merits and demerits of different types of EPON networking schemes. In addition, quantification is given for the EPON networking power consumption, so as to obtain a complete EPON combining scheme which is combined with specific examples to validate, and finally realize that the EPON technology has a certain application value because it is in line with various indicators after application in transmission line video monitoring.
\end{abstract}

Keywords: EPON technology; video monitoring; transmission line; application

\section{INTRODUCTION}

In recent years, with the development of economy and technology, China's power demand gradually increases and it causes a short supply. With an increase in transmission lines, the safety problems are also gradually exposed. To prevent natural or man-made damages to the lines has become an important task for the electric power sector. Thus, the transmission line video monitoring is very necessary.

At present, the application for EPON technology has matured in foreign countries, which has reached a high modular and commercial development level. Domestic operators also have applied for such technology on a large scale. However, the current domestic application fields are mainly concentrated in the distribution network. In recent years, people attach great importance to the video monitoring technology which is widely used in the transmission line. At present, compared with the wireless communication mode, the country pays more attention to the wireless communication system with flexible networking, good stability and low costs. As for the access network technology, EPON has absolute advantages in the remote control, power on and outage, and gradually becomes a "leader" in the distribution network field. Its application prospect is very broad due to its stability and efficiency.

This paper has analyzed EPON networking schemes and optical power consumption, and finally focused on the research about practical application of EPON technology in transmission line video monitoring.

\section{DESIGN PRINCIPLE OF TRANSMISSION LINE VIDEO MONITORING}

The main task of transmission lines is to supply electric energy, which is an extremely important part in realization of long-distance transmission of electric energy. The operating condition of transmission lines directly determines the efficiency of the power system. After transmission by the transmission lines from the power plant, the electricity transported to the load center requires to withstand the test of various risks. Therefore, faced with such a complex environment, it is extremely important to protect transmission line video monitoring.

Considering the construction operation and other factors, centered on low costs and high quality, and based on the principle of reliability, feasibility, rationality and practicability, the transmission line video monitoring is mainly summarized as follows:

(1) Advancement and maturity of the technology

Under the premise of the function of transmission lines, video monitoring technology should be advanced, which can play a leading and exemplary role in the whole power system; in term of the selection of system monitoring technology, the technological priority occupies an important position in the application of industry, and ensures smooth operation of the system, so as to achieve stable protection of the power system.

(2) Expansibility and rationality of the framework

The possibility of producing bottleneck problems on various subsystems in an overall system can be reduced by using mature technical framework. In terms of the equipment selection, it is necessary to 


\section{MATEC Web of Conferences}

leave room for future product upgrading, so there is a need to comprehensively consider the expansibility of various subsystems

(3) Timeliness and reliability of the system

In the transmission line video detection, there is an inseparable relationship between the efficiency of power system and the security and stability. To ensure safe operation of the transmission lines, the design of transmission line system must be ensured to have certain reliability, and effectively protect video detection, thus realizing video transmission.

\section{APPLICATION OF EPON IN VIDEO DETEC- TION}

\subsection{EPON networking schemes}

As a kind of mature optical access network technology, Ethernet Passive Optical Network (hereinafter referred to as "EPON") has been widely used in the field of electric power supply. ONU (Optical Network Unit), ODN (Optical Distribution Network) and OLT (Optical Line Terminal) are main components of EPON. Wherein, ONU provides a user-side interfaces and an optical interface between ODN and users, which realizes signal processing and maintenance management of the user equipment; the electrical interface is located at user side, while the optical interface is located at the side of ONU network. OLT provides a variety of business with transmission means, and reduces the tight coupling of management between the equipment, and its main purpose is to provide optical interfaces between ODN and business network. EPON has the network flexibility, which has a superior adaptability to the direction of optical cable. The main compositions of reducing costs are tree network structure, star network structure and ring network structure. The main structures are as follows:

(1) Ring structure

Under a certain circumstance, the ring structure can usually improve network reliability, which is a special kind of chain structure with a great practical value.

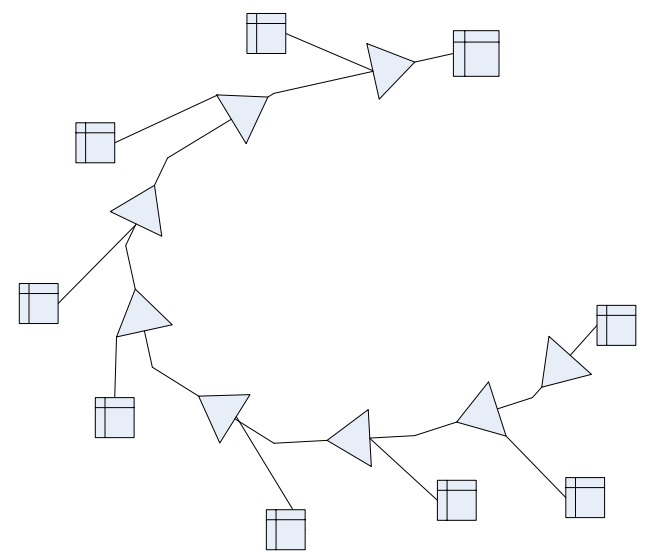

Figure 1. Ring OND structure chart

\section{(2) Chain structure}

The chain structure is connected in series by unbalanced optical branching devices, which transmits signal sent by ONU to OLT after inserting into the optical bus, thus facilitating OLT detected by the whole optical lines to send signals. In this way, it is possible to introduce additional losses, thereby increasing the optical power consumption.

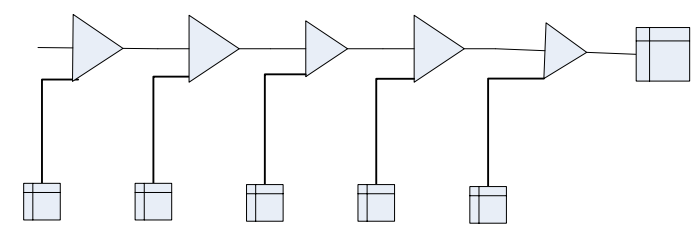

Figure 2. Chain OND structure chart

(3) Tree structure

The most common structure type in EPON is the tree structure, which is a basis structure configured from point to multipoint. Typical ODN structure is shown in Figure 3. This structure chart can transmit outbound signal to many users through the optical branching device, and transmit to OLT after confluence with the uplink signals.

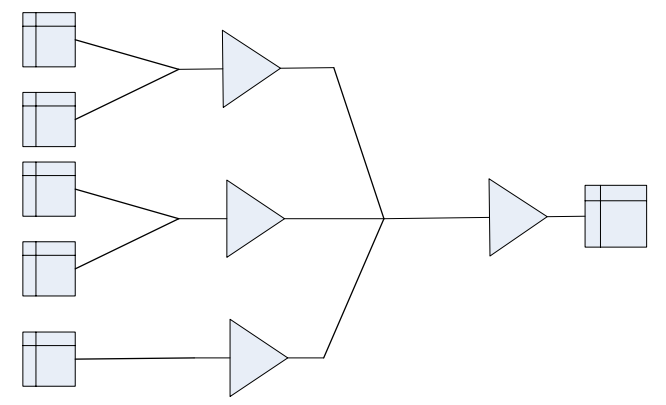

Figure 3. Tree OND structure chart

\subsection{Analysis of optical power consumption}

Reasonable configuration of ODN is significantly important in the design of progressive networking. The branch optical fiber, optical splitter and main optical fiber jointly constitute ODN. Only when the optical power accepted by ONU is within the range of ONU optical interface, normal transmission of optical signals between OLT and ONU can be achieved, so it is extremely important to analyze the optical power consumption. In ODN network with multistage spectral cascade, in order to display the series of multiple spectral effects, a non-uniform distributed optical splitter which is $1: 2$ is generally adopted to realize networking. Therefore, in the design of multistage non-uniform PDN network, it requires precise calculation to determine the power ratio of uniform optical splitter and spectral series. 
EMME 2015

Table 1. Parameter list of power extraction of non-uniform optical splitter

\begin{tabular}{llllll}
\hline Optical splitting ratio & $40: 60$ & $30: 70$ & $20: 80$ & $10: 90$ & $5: 95$ \\
Maximum allowable deviation range $(\mathrm{dB})$ & 0.4 & 0.4 & 0.4 & 0.4 & 0.4 \\
Typical value of insertion loss $(\mathrm{dB})$ & $4.4 / 2.6$ & $5.6 / 1.9$ & $7.4 / 1.3$ & $10.4 / 0.9$ & $11.8 / 0.6$ \\
\hline
\end{tabular}

Currently, in the EPON system, PX02 optical module has been widely used. The actual engineering practice and series indicators of optical communications show that, the maximum optical power from OLT to ONU is normally set as $26 \mathrm{~dB}$. By the use of this optical module, the maximum optical power at the opposite direction can be set as $24 \mathrm{~dB}$. The power consumption of non-uniform optical splitter is shown in Table 1.

1. Power consumption at the interface

The component mostly used in the optical passive device is an optical fiber splice. It generally forms a passive device by two optical fibers, which is mainly divided into the fusion fixture splice with the power attenuation of $0.2 \mathrm{Db} /$ splice, and the moving splice with the power attenuation of $0.5 \mathrm{Db} /$ splice.

2. Inherent power consumption of optical fiber

The consumption in the process of optical signal transmission is mainly because the impurity of optical materials has a stronger optical absorption capacity. In the process of transmission with $1,490 \mathrm{~nm}$ of wave length, the attenuation coefficient is weakened based on $0.2 \mathrm{Db} / \mathrm{km}$, while the transmission with $1,310 \mathrm{~nm}$ of wave length is weakened based on $0.36 \mathrm{Db} / \mathrm{km}$.

3 . Surplus degree of the system

After putting into use of the optical fiber, its aging phenomenon and surrounding environment will have a certain impact on power consumption. On this basis, the concept on surplus degree of the optical fiber is introduced, and a certain budget is left for the future potential power losses, which is generally set as $3 \mathrm{~dB}$ in engineering. Therefore, the formula of a total power budget of ODN optical link is as follows:

$P=\sum L_{i}+\sum M_{i}+\sum N_{i}+\sum K_{i}+G$

Where: $G$ is the power surplus degree of the system; $\mathrm{Ki}$ is the insertion consumption of optical path; $\mathrm{Ni}$ is the power consumption of the splice; $\mathrm{Mi}$ is the power extraction of moving slice; $\mathrm{Li}$ is the power attenuation of each section of optical fiber.

In this design of multistage non-uniform ODN network, the maximum spectral series of the system is calculated by the maximum one. For the selection of optical power distribution ratio, each branch in different programs should be calculated, so as to select the best program. For a typical multistage uniform optical OND network, the length of optical splitter at each segment is respectively $2.6 \mathrm{~km}, 2.4 \mathrm{~km}, 2.2 \mathrm{~km}, 2.1 \mathrm{~km}$, $2.2 \mathrm{~km}, 2.3 \mathrm{~km}$ and $2.6 \mathrm{~km}$. The splice of each section of optical fiber is a moving splice with the power ratio of $20: 80$.

According to the above conditions, the dissipation of optical fiber lines at the uplink direction is as follows: $\sum L_{i}=0.22 \times(2.6+2.4+2.2+2.1+2.2$

$+2.3+2.6)=3.608(d B)$

The power dissipation on moving splice of the optical fiber is:

$\sum M_{i}=7 \times 2 \times 0.5=7(d B)$

The dissipation on fusion node of the optical fiber is:

$\sum N_{i}=7 \times 0.2=1.4(d B)$

The insertion loss of optical splitter is:

$\sum K_{i}=7 \times 1.3=9.1(d B)$

From the above, the total power consumption of the lines is as follows:

$P=5.904+7+1.4+9.1=23.404(d B)$

According to the above statement, the surplus degree of the optical fiber is $3 \mathrm{~dB}$, and the uplink optical power is $26.4 \mathrm{~dB}$, far exceeding the prediction of the maximum optical power. Therefore, such a program requires to be amended. The power dissipation can be adjusted by the use of optical splitter which is 5:95, so as to achieve the requirements of indicators.

According to the above conditions, the dissipation of optical fiber lines at the down direction is as follows:

$\sum L_{i}=0.22 \times(2.6+2.4+2.2+2.1+2.2$

$+2.3+2.6)=3.608(d B)$

The power dissipation on moving splice is:

$\sum M_{i}=7 \times 2 \times 0.5=7(d B)$

The power dissipation on the nodes of optical cable is:

$\sum N_{i}=7 \times 0.2=1.4(d B)$

The insertion loss of optical splitter is:

$\sum K_{i}=7 \times 1.3=9.1(d B)$

From the above, the total power consumption of the lines is:

$P=3.608+7+1.4+9.1=21.108(d B)$ 
MATEC Web of Conferences

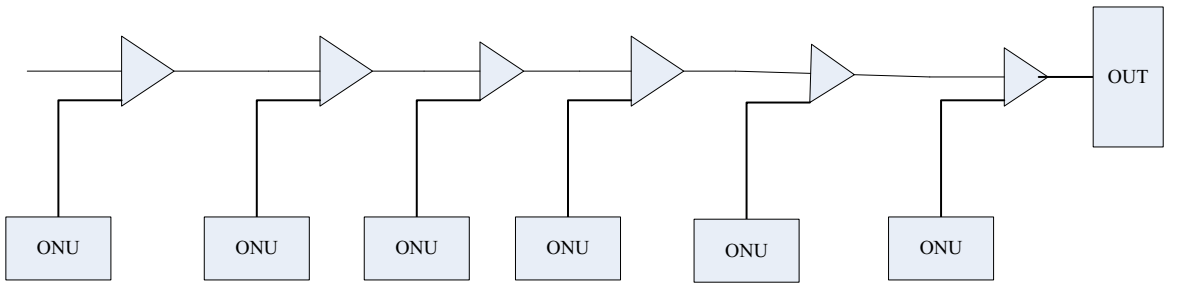

Figure 4. Program 1

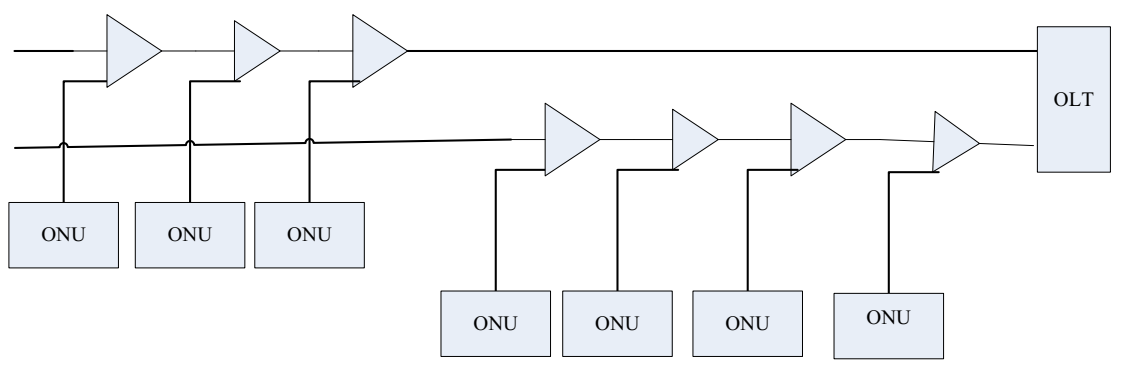

Figure 5. Program 2

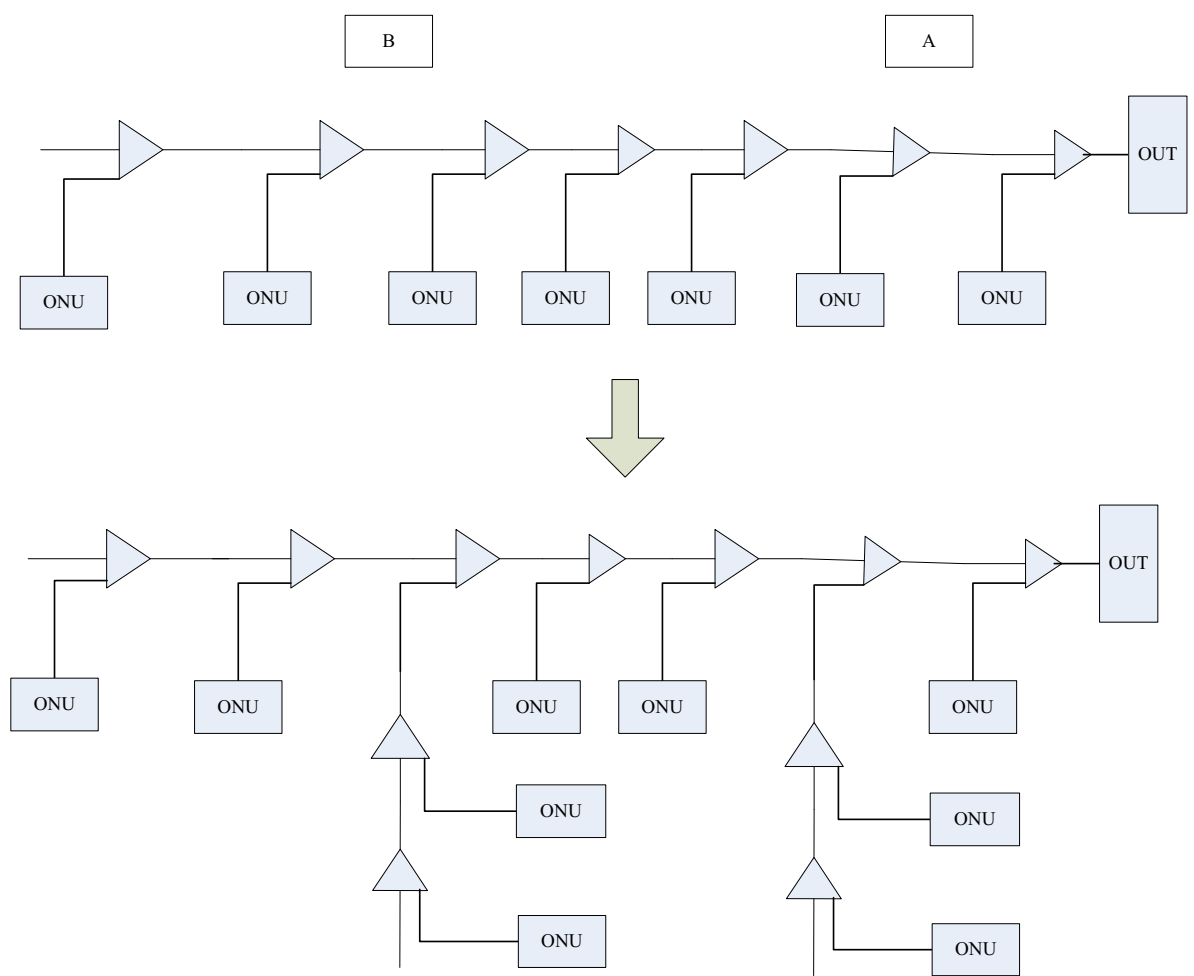

Figure 6. Schematic diagram of network expansion.

According to the above statement, the surplus degree of the optical fiber is $3 \mathrm{~dB}$, so the optical power budget at the down direction is in line with the design requirements.

ODN network in EPON system is usually designed as multistage network mainly due to the chain network structure of transmission lines. Different proportions of optical splitter used herein will eventually transit to 1: 1 , so that the optical power dissipation of each ONU gradually becomes the same. Considering from 
simplifying the network, reducing the series of optical splitter can provide more space for future expansion of the network and effectively improve the reliability of ODN network. Figures 4 and 5 respectively show two different design schemes: the cascade scheme adopts one four-grade optical splitter, one three-grade optical splitter and two PON interfaces; the other cascade scheme adopts one seven-grade optical splitter and one PON interface.

Compared with the second scheme, the first scheme is added with a one-core backbone optical fiber, which can make the optical power rate in an entire network surplus, and provide the possibility for greater development space in the future.

With the continuous development of smart power grid, the line construction and protection in the power industry are also continuously upgrading. Therefore, faced with planning of the power grid in the future, there is also a need to consider whether the network communication possesses a surplus degree after rent. Figure 6 is a schematic diagram of network expansion.

To plan the power grid construction in the future, if there are expansion needs at the point of $\mathrm{A}$ and $\mathrm{B}$, in the construction of ODN network, there is a need to set aside much enough optical power budget at these two points. To enhance the surplus space of the network, generally, there is a need to enhance the backbone fiber core. Through setting aside sufficient optical power, the operating rate can be significantly increased, while the costs can be significantly reduced.

\subsection{EPON networking schemes}

EPON networking scheme is proposed based on the construction conditions of the substation, transmission line tower, and actual needs of video monitoring: The chain topology structure is a major structural model adopted by EPON optical distribution network. Generally, the distance between each ONU is within the range of 2 to $4 \mathrm{~km}$, which belongs to flexible distance. OLT mainly collects the transmission line video monitoring information within $20 \mathrm{~km}$.

To apply for EPON access networking technology in the transmission line and substation video monitoring can make a higher level of dispatching center use the existing power communication network, and realize data collection, transmission and processing in the entire video monitoring system.

\section{APPLICATION OF EPON TECHNOLOGY IN THE TRANSMISSION LINE VIDEO MONI- TORING}

According to the data provided by the communication automation center of Liaoning Electric Power Co., Ltd., the distance between power cable connector boxes $(\mathrm{a}, \mathrm{b}, \mathrm{c}, \mathrm{d})$ is respectively $4.2 \mathrm{~km}, 4.5 \mathrm{~km}$ and $3.6 \mathrm{~km}$. The distance between the substation and connector box is $3.4 \mathrm{~km}$. The selection of optical splitter is shown in Table 2:
Table 2. Parameters of optical splitter

\begin{tabular}{|c|c|c|c|c|}
\hline Location of optical splitter & $\mathrm{a}$ & $\mathrm{b}$ & $\mathrm{c}$ & $\mathrm{d}$ \\
\hline $\begin{array}{l}\text { Typical value of insertion } \\
\text { loss (dB) }\end{array}$ & $10.4 / 0.9$ & $7.4 / 1.2$ & $5.5 / 1.9$ & $4.4 / 2.5$ \\
\hline $\begin{array}{l}\text { Spectral ratio by the use of } \\
\text { optical splitter }\end{array}$ & $10: 90$ & $20: 80$ & $30: 70$ & $40: 60$ \\
\hline
\end{tabular}

The power dissipation at the uplink direction is calculated as follows:

The dissipation on the lines of optical fiber is:

$\sum L_{i}=(4.2+4.5+3.6+4.3) \times 0.36=5.98(d B)$

The dissipation on moving splice is:

$\sum M_{i}=4 \times 2 \times 0.5=4(d B)$

The dissipation on fusion node of the optical cable is:

$\sum N_{i}=0.2 \times 4=0.8(d B)$

The insertion loss of optical splitter is:

$\sum K_{i}=2.6+1.9+1.2+0.9=6.5(d B)$

The total power consumption of the lines is:

$P=6.5+0.8+4+5.97=17.3(d B)$

Combined with the surplus degree of the optical fiber, the power budget at the uplink direction should be smaller than the predetermined $24 \mathrm{~dB}$;

The power dissipation at the down direction is calculated as follows:

The dissipation on the lines of optical fiber is:

$\sum L_{i}=(4.2+4.5+3.6+4.3) \times 0.22=3.65(d B)$

The dissipation on moving splice is:

$\sum M_{i}=4 \times 2 \times 0.5=4(d B)$

The dissipation on fusion node of the optical cable is:

$\sum N_{i}=0.2 \times 4=0.8(d B)$

The insertion loss of optical splitter is:

$\sum K_{i}=2.6+1.9+1.2+0.9=6.5(d B)$

The total power consumption of the lines is:

$P=6.5+0.8+4+3.625=14.9(d B)$

Combined with the surplus degree of the optical fiber, the power budget at the down direction should be much smaller than $26 \mathrm{~dB}$, indicating that the design is in line with requirements. Through the above analysis, 


\section{MATEC Web of Conferences}

EPON splitting ratio can be fully adopted to meet the consumption problems on the optical cable networking function.

\section{CONCLUSION}

This paper carries out comparative analysis of EPON networking schemes and structure types with different features; second, this paper analyzes the optical power consumption, and determines the power ratio of uniform optical splitter and spectral series in multistage non-uniform PDN network through precise calculation, and also carries out comparative analysis of the design of different practical networking schemes. The result is that the increase of one-core backbone optical fiber may make the optical power rate in an entire network surplus. Finally, combined with the previous theoretical analysis, this paper focuses on the research on the application of EPON technology in the transmission line video monitoring, and proves that such technology has practical application value, thus playing certain guidance significance in the development of the field in the future.

\section{REFERENCES}

[1] Zhao Jianqing, Yao Yao, Qiu Wanhui, Tang Jinrui, Liu Hongjie, Li Zhenyu, Chen Xu, Xie Jing \& Yin Xianggen. 2013. Research on smart early warning system based on transmission line online inspection system. Power System Protection and Control, 23: 50-55.

[2] Shigemitsu Okabe. \& Toshihiro Tsuboi. 2011. Analysis of Aspects of Lightning Strokes to Large-sized Transmission Lines. IEEE Transactions on Dielectrics and Electrical Insulation. 18(1):180-1189.
[3] Zhou Xin, Zhu Lan. \& Wu Jiang. 2011. Research on Networking of EPON in smart communication system of power distribution and utilization network. Design Technology of Posts and Telecommunications. 1: 52-59.

[4] Zhao Jianqing, Tang Jinrui, Qiu Wanhui, Yao Yao, Liu Hongjie, Li Zhenyu, Qian Yimin, Xie Jing. \& Yin Xianggen. 2013. Online inspection system of power transmission line based on optical fiber composite overhead ground wire communication. Guangdong Electric Power, 12: 12-18

[5] ZHANG Hao, BU Xian-de. \& GUO Jing-hong. 2010. Application of EPON technology in power consumption information collection system. Telecommunications for Electric Power System, 31(7): 40-46.

[6] Chen Jieming. 2011. Research on GOOSE-based $10 \mathrm{kV}$ simple busbar protection and application. Power system automation, (4): 94-98

[7] Yin Hua. \& Wang Fa. 2010. Application of wireless Mesh in transmission line video monitoring. Power System Communication. 21 (215): 40-45

[8] W. Zhang, \& M. Branieky. 2001. Stability of Networked Control Systems. IEEE Control Systems Magazine. 21

[9] Luo Jianbin. 2013. Research on high-voltage transmission line icing condition monitoring based on optical fiber sensing technology. South China University of Technology.

[10]JIAO Xiao-bo, ZHOU Ya, \& LI Hong-jun. 2010. Application and development of OPGW cables in power optical transmission network. Study on Optical Communications, (4): 44-50.

[11]Qian Yimin. 2013. Research on transmission line online inspection and monitoring new technology based on OPGW optical fiber communications. Huazhong University of Science and Technology. 